\title{
AN EXACT SEQUENCE IN DIFFERENTIAL TOPOLOGY
}

\author{
MORRIS W. HIRSCH \\ Communicated by Deane Montgomery, May 12, 1960
}

1. Introduction. The purpose of this note is to describe an exact sequence relating three series of abelian groups: $\Gamma^{n}$, defined by Thom [3]; $\theta^{n}$, defined by Milnor [1]; and $\Lambda^{n}$, defined below. The sequence is written

$$
\cdots \rightarrow \Gamma^{n} \stackrel{j}{\rightarrow} \theta^{n} \stackrel{k}{\rightarrow} \Lambda^{n} \stackrel{d}{\rightarrow} \Gamma^{n-1} \rightarrow \cdots .
$$

We now describe these groups briefly.

To obtain $\Gamma^{n}$, divide the group of diffeomorphisms of the $n-1$ sphere $S^{n-1}$ by the normal subgroup of those diffeomorphisms that are extendable to the $n$-ball. See [2] for details.

The set $\theta^{n}$ is the set of $J$-equivalence classes of closed, oriented, differentiable $n$-manifolds that are homotopy spheres. If $M$ is an oriented manifold, let $-M$ be the oppositely oriented manifold. Two closed oriented $n$-manifolds $M$ and $N$ are $J$-equivalent if there is an oriented $n+1$-manifold $X$ whose boundary is the disjoint union of $M$ and $-N$, and which admits both $M$ and $N$ as deformation retracts. We denote the $J$-equivalence class of $M$ by [M]. If [M] and [N] are elements of $\theta^{n}$, their sum is defined to be [M\#N], where [ $\left.M \# N\right]$ is obtained by removing the interior of an $n$-ball from $M$ and $N$ and identifying the boundaries in a suitable way. Details may be found in $[1]$.

The group $\Lambda^{n}$ is defined analogously using combinatorial manifolds. Instead of the interior of an $n$-ball, the interior of an $n$-simplex is removed. If $M$ is a combinatorial manifold, we write $\langle M\rangle$ for its $J$ equivalence class.

2. The sequence. To define $k: \theta^{n} \rightarrow \Lambda^{n}$, we observe that every differentiable manifold $M$ defines a combinatorial manifold $\bar{M}$, unique up to combinatorial equivalence, by means of a smooth triangulation of $M$ [4]. We define $k[M]=\langle\bar{M}\rangle$.

Let $g: S^{n-1} \rightarrow S^{n-1}$ represent an element $\gamma$ of $\Gamma^{n}$. According to J. Munkres [2], there is a unique (up to diffeomorphism) differentiable manifold $V_{\gamma}$ corresponding to $\gamma$, such that $\bar{V}_{\gamma}=\bar{S}^{n}$. To obtain $V_{\gamma}$, identify two copies of $R^{n}-0$ by the diffeomorphism $x \rightarrow(1 /|x|) g(x /|x|)$. Here $R^{n}$ is Euclidean $n$-space and $|x|$ is the usual norm. The diffeomorphism class of $V_{\gamma}$ depends only on $\gamma$, and 
$V_{\gamma}$ is diffeomorphic to $V_{\delta}$ if and only if $\gamma=\delta$. We define $j: \Gamma^{n} \rightarrow \theta^{n}$ by $j(\gamma)=\left[V_{\gamma}\right]$.

To define $d: \Lambda^{n} \rightarrow \Gamma^{n-1}$, we proceed as follows. If $\langle M\rangle$ is an element of $\Lambda^{n}$, let $M_{0}$ be obtained from $M$ by removing the interior of an $n$-simplex. According to a recent result of A. M. Gleason, there is a differentiable manifold $N$ such that $\bar{N}=M_{0}$. By [2], $N$ is unique up to diffeomorphism, because $M_{0}$ is contractible. Since the boundary $\partial N$ of $N$ is combinatorially an $n-1$ sphere, there is a unique $\beta \in \Gamma^{n-1}$ such that $\partial N=V_{\beta}$. We define $d\langle M\rangle=\beta$. It can be shown that $\beta$ depends only on $\langle M\rangle$.

THEOREM. The sequence (1) is exact.

The proof will appear in a subsequent paper.

\section{BIBLIOGRAPHY}

1. J. Milnor, Differentiable manifolds which are homotopy spheres, Princeton University, 1959 (mimeographed).

2. J. Munkres, Obstructions to the smoothing of piecewise differentiable maps, Annals of Mathematics, to appear.

3. $\mathrm{R}$. Thom, Des variêtés triangulées aux varietés differentiables, Proceedings of the International Congress of Mathematicians, 1958, pp. 248-255.

4. J. H. C. Whitehead, On $C^{1}$ complexes, Ann. Math. vol. 41 (1940) pp. 809-824.

The Institute for Advanced Study 